Journal of Machine Engineering, 2020, Vol. 20, No. 3, 44-62

ISSN 1895-7595 (Print) ISSN 2391-8071 (Online)

Received: 14 January 2020 / Accepted: 07 March 2020 / Published online: 25 September 2020

Double-Ballbar, fast performance test, volumetric motion accuracy, multi-axis machine tools

\author{
Bernd KAUSCHINGER ${ }^{1 *}$ \\ Christian FRIEDRICH ${ }^{1}$ \\ Ruiqing $\mathrm{ZHOU}^{1}$ \\ Steffen IHLENFELDT ${ }^{1}$
}

\title{
FAST EVALUATION OF THE VOLUMETRIC MOTION ACCURACY OF MULTI-AXIS MACHINE TOOLS USING A DOUBLE-BALLBAR
}

\begin{abstract}
The proof of manufacturing accuracy requires continuous verification and crosscheck of the motion accuracy of machine tools. Machining in 5 to 6 axes intensifies the problem of measurement and evaluation of volumetric motion accuracy in up to 6 degrees of freedom (DOF) in the whole workspace. Although, there are many known, even standardized, measuring methods, they are either expensive, time-consuming, not applicable in an operational state of the machine under shop floor conditions, or their significance is limited to only 1 or 2 feed-axes. Appropriate methods to be run regularly, fast and cost-efficient by the machine operator as a performance test are still desired. The article presents a new approach that meets these requirements. It is based on a Double-Ballbar (DBB) with enlarged measuring range and volumetric measuring paths of up to 6 DOF with all feed-axes moving simultaneously during continuous measurement, instead of plane circular paths according to ISO 230-4. After an explanation of the proposed method, the developed DBB device is introduced, including its mechanical and sensor design, the data interface, and results of experimental investigations on the measuring accuracy. Furthermore, relevant problems regarding the design, optimization, and programming of appropriate 6 DOF measuring paths are discussed and experimental results are presented that show the advantage compared to other measuring paths.
\end{abstract}

\section{INTRODUCTION}

A relevant influence on the manufacturing accuracy at the workpiece is the motion accuracy of the machine tool. Typically, it becomes exemplary effective not until the operational state, due to manufacturing, assembly and setup of the machine tool. Moreover, depending on installation as well as environmental and operating conditions, properties and states of machine parts and components, and thus, the motion accuracy, change over time for various reasons, such as tool or workpiece change, run-in, wear, overload, collisions, lubrication or transient behaviour. In order to ensure compliance with the accuracy requirements and to

\footnotetext{
${ }^{1}$ Institute of Mechatronic Engineering, Chair of Machine Tools Development and Adaptive Controls, TU Dresden, Germany

*E-mail: bernd.kauschinger@tu-dresden.de https://doi.org/10.36897/jme/119678
} 
avoid production faults with high subsequent costs, a regular evaluation of the motion accuracy is required. In this context, there are two purposes of evaluation measurements:

1. The motion accuracy is checked and validated for compliance with the accuracy requirements. Rather integral instead of detailed information is sufficient for this, which usually can be acquired by so-called performance tests, e.g. [1,2]. Suitable measurements must deliver trustable statements of rather qualitative nature as a basis for decisions, if additional measures, efforts or investments will be necessary or not. For economical reasons, only low effort is accepted to derive statements for decision support.

2. If accuracy requirements are not met, then additional measures to improve motion accuracy are necessary. Among others, a variety of model based correction approaches is used, which requires the determination or calibration of model parameters [3-5].

3. This is based on exemplary measurements of the motion accuracy, which are of quantitative nature, in principle. Here, usually a higher effort is needed and accepted to get calibration values that essentially determine the quality of correction results.

Particular evaluation scenarios allocated in the life cycle of machine tools are exemplarily listed in Table 1 . They preferably consider the machine tool in an operational state and differ in both the required evaluation statements and the accepted effort.

Table 1. Scenarios for measurement based evaluation of the motion accuracy of machine tools

\begin{tabular}{|c|c|c|c|c|}
\hline & Scenario & Evaluation statements & $\begin{array}{l}\text { Relevant } \\
\text { for ... }\end{array}$ & $\begin{array}{c}\text { Accepted } \\
\text { effort }\end{array}$ \\
\hline \multirow{2}{*}{ 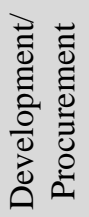 } & $\begin{array}{l}\text { comparison } \\
\text { of variants }\end{array}$ & $\begin{array}{l}\text { behaviour of the same machine } \\
\text { in different design states }\end{array}$ & manufacturer & medium \\
\hline & $\begin{array}{l}\text { machine } \\
\text { selection }\end{array}$ & $\begin{array}{l}\text { behaviour of similar machines } \\
\text { from different manufacturers }\end{array}$ & machine user & low \\
\hline \multirow{2}{*}{ 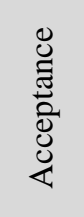 } & $\begin{array}{l}\text { quantitative } \\
\text { assessment }\end{array}$ & $\begin{array}{l}\text { proof of accuracy at delivery via a set of accuracy } \\
\text { parameters, e.g. positioning deviations }\end{array}$ & $\begin{array}{l}\text { manufacturer, } \\
\text { machine user }\end{array}$ & high \\
\hline & $\begin{array}{l}\text { qualitative } \\
\text { assessment }\end{array}$ & $\begin{array}{l}\text { proof of accuracy at delivery, } \\
\text { e.g. test pieces, circular tests }\end{array}$ & $\begin{array}{l}\text { manufacturer, } \\
\text { machine user }\end{array}$ & low \\
\hline$\cong$ & $\begin{array}{l}\text { correction } \\
\text { model setup }\end{array}$ & $\begin{array}{l}\text { specific error values, e.g. spindle pitch error, cross } \\
\text { compensation error, volumetric grid error }\end{array}$ & $\begin{array}{l}\text { manufacturer, } \\
\text { machine user }\end{array}$ & high \\
\hline \multirow{2}{*}{ 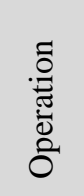 } & $\begin{array}{l}\text { machine } \\
\text { diagnosis }\end{array}$ & $\begin{array}{l}\text { machine behaviour after a crash or repair, } \\
\text { cross-check of motion accuracy }\end{array}$ & machine user & low \\
\hline & repeated test & $\begin{array}{l}\text { behaviour change over life time, correction model } \\
\text { verification, accuracy check in production breaks }\end{array}$ & machine user & very low \\
\hline
\end{tabular}

Current trends in production towards individual workpieces with greater shape complexity and reduced batch sizes (up to a quantity of one) lead to an increasing use of universal machining centers, up to simultaneous 5-axis machining [6]. The larger number of interpolated feed-axes results in an even greater variation of the already position dependent machine behavior [7] in the workspace. Moreover, besides a high repeatability also a high 
absolute accuracy of the machine tool in its whole workspace is demanded. This intensifies the problem of volumetric motion accuracy, including its measurement and evaluation in up to $6 \mathrm{DOF}$. In future, a more frequent validation of volumetric motion accuracy will be required to support decisions about continued production or necessary maintenance or repair. Scenarios of fast machine diagnostics and repeated measurements that are carried out by the machine operator itself and limit unproductive machine downtime will become more important. However, usually it may be more expensive than necessary to use measuring methods that are suitable for calibration measurements for this, even though there are also attempts for uniform measurements, e.g [8]. Suitable measuring methods to deliver statements for decision support must meet the following requirements for fast performance tests:

- fast, simple and robust measuring procedure,

- applicable in the operational state of the machine,

- measuring accuracy better than manufacturing accuracy required,

- low effort for installation, setup and execution of the measurements,

- representative coverage of the workspace in all relevant DOF (up to 6),

- low investment and operating costs of the measuring system.

Although, there is a variety of measuring systems and methods for the evaluation of motion accuracy of machine tools used so far, none really fulfils all of the requirements. This motivated the development of the measuring method proposed in this article.

Due to its large measuring range combined with a high measuring accuracy and resolution laser based measurement is advantageous for calibration and correction approaches. Volumetric errors in the workspace are captured by 3D laser trackers or by multi-lateration with 1D lacer tracers or 1D laser ballbars. Axis specific errors are captured with 1D to 6D laser interferometers (Fig. 1). MultiLine laser systems can capture absolute distances of multiple measuring lines in up to 32 channels. However, since laser based equipment is not least one of the most expensive one, it is rather in economic for a fast validation of motion accuracy. Moreover, setup and use are time-consuming in many cases.

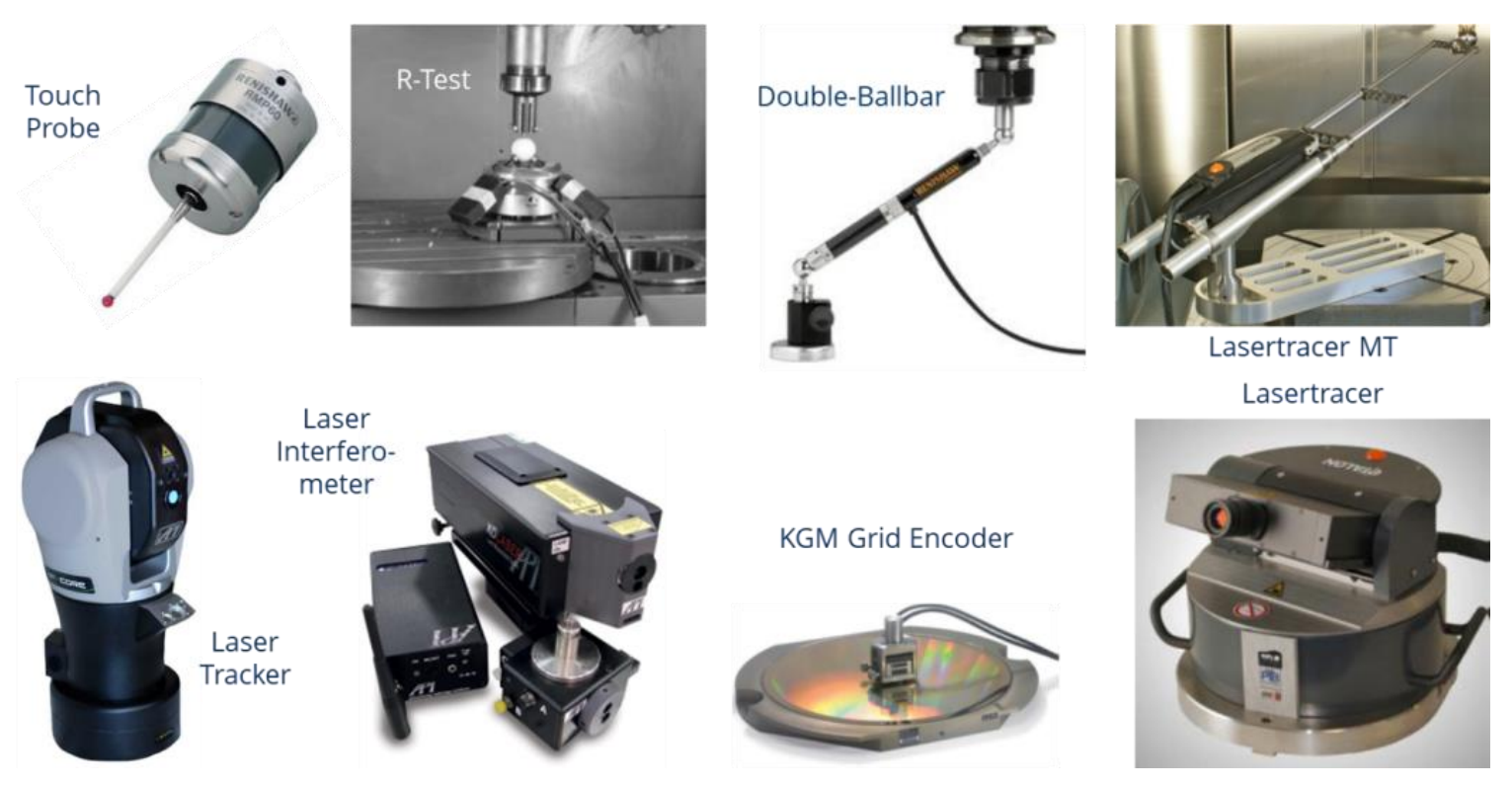

Fig. 1. Examples of measuring systems for the evaluation of machine accuracy 
There are also measuring systems and methods, that base on specific reference artifacts, like prismatic references, precision balls, ball plates or cross grids. Measuring systems like linear gauges, touch probes, QuickCheck, R-Test or cross grid encoders (KGM) are used to measure geometric deviations with reference to these artifacts (Fig. 1). However, the statements derived are either limited to single poses or axes in the workspace or very timeconsuming for a larger number of poses or axes. Moreover, the precision requirements for the artifacts are high to be suitable as reference, which usually makes them quite expensive.

Another common approach is the machining of test workpieces (Fig. 2) and their subsequent evaluation by a coordinate measuring machine (CMM). However, this procedure is time-consuming and requires additional material expenses (e.g. work pieces, auxiliary material) as well as additional survey at a CMM. The derivable statements are limited to the workspace volume covered by the test workpiece and current machining conditions. Due to the effort, machining of test workpieces is rather suitable for final acceptance tests or for the optimization of manufacturing processes for large batch sizes but not for a fast validation of motion accuracy.

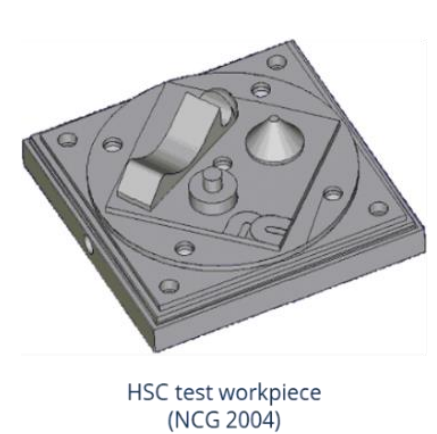

(NCG 2004)

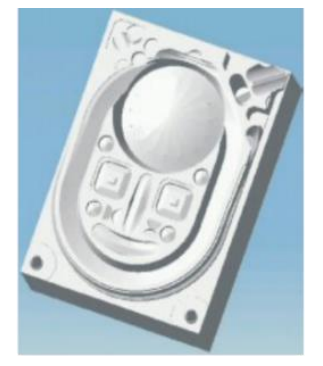

5-axis test workpiece (NCG 2005)

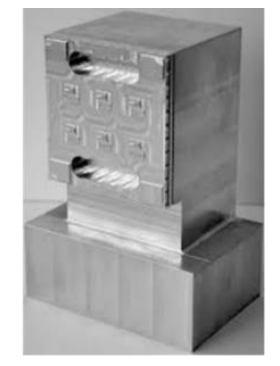

test workpiece for thermal behavior (SFB/TR96)

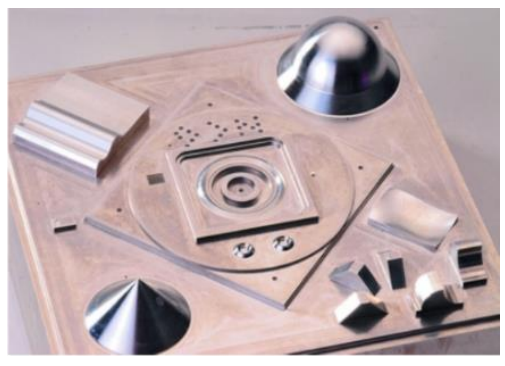

5-axis test workpiece for micro-machining (NCG 2007)

Fig. 2. Examples of test workpieces for the evaluation of the motion behaviour of NC machine tools

DBBs are used as measuring devices for various tasks. Besides their main application for circular tests according to ISO 230-4 [1] for the evaluation of pairs of feed-axes using circular paths, DBBs are also used for the calibration of rotary axes in five-axis machine tools [5], for the assessment of elasto-static or thermal behaviour of machine tools [9-11] or for the kinematic calibration of non-linear kinematic structures like parallel kinematic machines (PKM) [12] or industrial robots (IR).

DBB measurements fulfil nearly all of the requirements for fast performance tests. However, they lack a representative coverage of the workspace in up to $6 \mathrm{DOF}$, so far. But, by enlarging the measuring range and using volumetric measuring paths this obstacle can be overcome. To derive the idea of an enhanced DBB design and of the proposed measuring approach, the general design of a DBB, its basic measuring principle and relevant consequences are summarized in Section 2 first. The design of an inexpensive DBB device with enhanced measuring range is depicted in Section 3. Comparative investigations on the measuring accuracy and repeatability of the developed DBB are shown in Section 4. Challenges and approaches for the design of volumetric measuring paths are outlined in Section 5, while Section 6 shows advantages of 6 DOF measuring paths with results of DBB measurements. Section 7 finally closes the article with a summary and outlook. 


\section{TOWARDS A DBB BASED MEASUREMENT OF VOLUMETRIC ACCURACY}

The idea of the DBB is not new. First papers [13] and already expired patents $[14,15]$ date back to 1982. A DBB is a specific measuring device made of a telescopic "bar" that mechanically connects two balls at its ends and contains a linear position sensor (Fig. ). The balls are made of ferro-magnetic material (steel) and are attached magnetically into a specific three-point fixture, each (Fig. 3, right). These magnetic fixtures enable an easy attachment and detachment of the balls with a very good repeatability of the ball center position. Each ball in its fixture makes up a ball-and-socket joint with (ideally) constant center position and 3 rotational DOF. Depending on the ball quality the uncertainty of a ball center position in its fixture is very low, e.g. below $1 \mu \mathrm{m}$ for precision balls of grade G5. One fixture is mounted on the base or table of the machine tool, for the so-called fixed ball, while another one is mounted on a tool holder in the machine tool's spindle, for the so-called "moved ball".
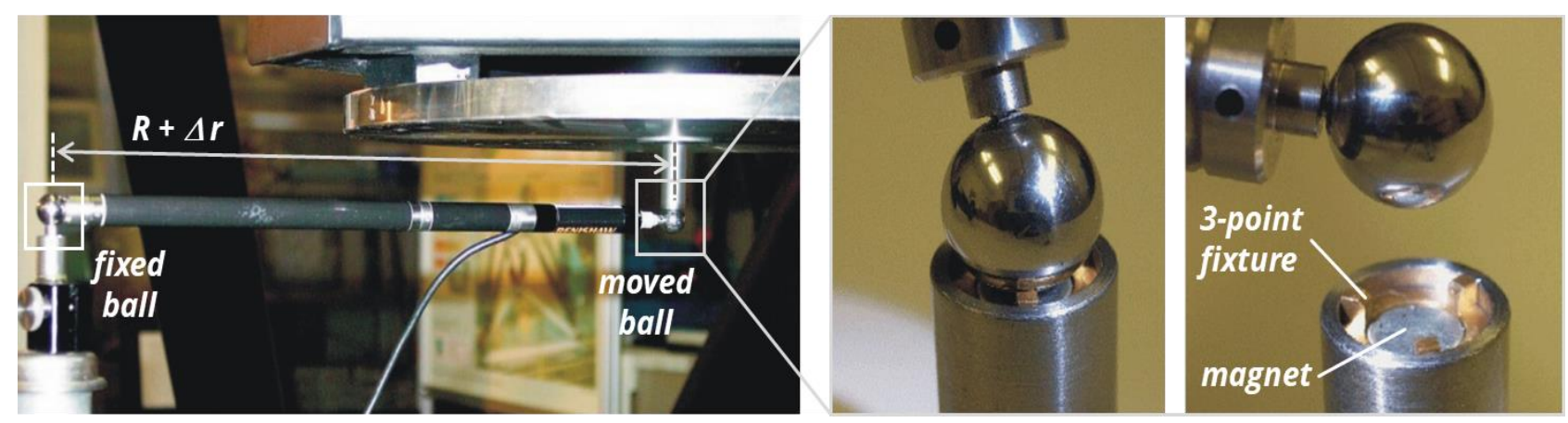

Fig. 3. Double-Ballbar (Renishaw QC10) with magnetic three-point fixtures

The measuring principle of the DBB is quite simple. The balls are either positioned or moved relative to each other. This is usually carried out by a controlled motion of the machine tool and requires the balls in their sockets to act as spherical joints. The nominal ball positions are specified in the motion command or the NC program. In an ideal machine tool the actual ball distance would correspond to the distance of the nominal ball positions. However, this is not possible in reality [9], since the machine tool's assemblies always carry out a relative motion in 6 DOF, regardless of the number of feed-axes involved [16]. Hence, the actual distance will always deviate from the nominal one, and the distance change is recognized and measurable by the DBB. Depending on the sensor system, measuring resolutions of below $1 \mu \mathrm{m}$ and measuring accuracies of around $1 \mu \mathrm{m}$ are typical in this context.

A single DBB measuring values is the translational part of the 6 DOF motion error at the according ball positions that acts along the respective measuring direction, which is spanned by the connecting line of the ball's centers. This way, a DBB projects the motion error from the 6-dimensional space onto a 1-dimensional subspace, which implies a loss of information about the motion error. In the literature, this aspect is also named "partial pose measurement".

However, DBB measurements can be seen equivalent to the machining of a spherical test workpiece by 3 to 6-axis-milling with a ball-end mill (Fig. 4). Here also only 1 out of 
$6 \mathrm{DOF}$ of the motion error causes manufacturing errors at the workpiece, namely the one in the respective normal direction of the milled surface of the workpiece. This means an equivalent loss of information about the motion error, which generally also occurs on other workpiece shapes in 5-axis machining, e.g. the test workpieces in Fig. 2. Nevertheless, compared to the machining of test workpieces, DBB measurements can be carried out faster, no additional material is needed, less collisions or axis limits occur, less error effects influence the measu-rements (e.g. process loads, tool deflection, tool wear or coolant and chips) and no additional survey of the actual workpiece geometry after machining is needed. Moreover, depending on the DBB measuring range, workspace areas of more complex geometry than circles or spheres can be evaluated with a DBB, e.g. linear, prismatic or edged features (see also Fig. 2).
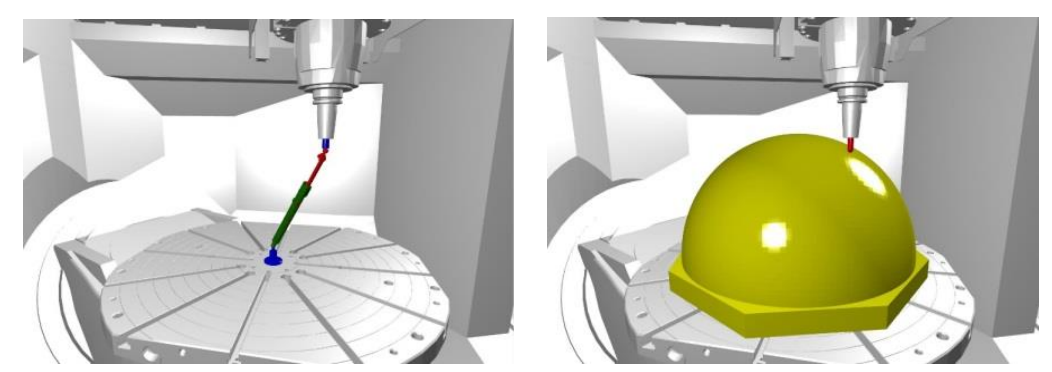

Fig. 4. DBB measurements (left) and equivalent machining of a spherical workpiece with a ball-end mill (right)

Although a single DBB value only contains information about the motion error at the respective pose (position and orientation) in the workspace, it is still possible to derive statements about motion error characteristics in a larger workspace area by combining many measuring values at different measuring poses and directions, respectively. With regard to the sphere-shaped workpiece in Fig. 4, this is equivalent to the evaluation of manufacturing errors at multiple surface points (e.g. using a touch probe, a CMM or a 3D scan). Depending on the distribution of the measuring poses, integral statements about the covered workspace area are derivable, e.g. based on minimal, maximal or average measured values. For the evaluation of motion accuracy, this will deliver practicable statements, since the extreme values of the motion errors inside a particular workspace volume are of major interest here, whereas their specific location often is of minor or even no relevance. In this context, the statements derived from DBB measurements are of rather integral and qualitative nature.

In the literature essentially three different variants to carry out DBB measurements are mentioned. A first variant is to move to a measuring pose, stop there, attach the $\mathrm{DBB}$, acquire the measuring value, detach the $\mathrm{DBB}$, and repeat this procedure successively at further measuring poses, e.g. [17]. This way, the poses either are connected successively by arbitrary paths or are represented by multiple balls in specific measuring artifacts. However, due to extensive manual interaction and a long measuring duration, this is only acceptable for a few poses.

A second, more advanced variant is to attach the DBB at only the first pose and detach it after the last one, and connect the single poses successively by circular arcs with constant radius around the fixed ball, e.g. [3]. In this way, the DBB can stay attached during the whole 
measurement and manual interactions are reduced to a minimum, but the connection paths are no longer arbitrary.

For both variants, the measured values are uniquely assigned to their measuring poses, since the motion is stopped for data capturing. However, for many measuring poses, the measuring duration is still high. Consequently, the third variant is to connect the measuring poses with a continuous motion path and to capture the measuring values continuously while moving (preferably with a high sampling rate of e.g. $250 \mathrm{~Hz}$ ) without stopping. This way, many measuring values are recorded in a short time at many poses with a high positional resolution. Depending on the measuring path length and speed, short measuring durations (minutes or even seconds), combined with a large number of measuring values (more than 10,000 samples per minute) are possible. As a result, the measuring duration is reduced to a minimum. For this reason, the third variant is preferred for fast performance tests, e.g. the one proposed here.

However, the captured DBB values are now uniquely assigned to their sampling time. Instead, an assignment to their respective pose of the measuring path is needed, which is far more challenging and requires additional measures. One of these measures is to use a measuring motion with constant speed and to guess the measuring poses based on the sampling times of the measured values. This approach is used in circular tests according to ISO 230-4. However, in practice the feed-axes are accelerated and decelerated nevertheless. Thus, the measuring speed must be low and additional leading and trailing motions are required. Alternatively, one could sample the actual positions of the feed-axes synchronously in addition and predict the measuring poses based on the sampling time of the actual positions and a forward kinematic model. This way, measuring speed can be increased and measuring duration reduced. However, adequate access to the actual axis positions must be available in the control with high sampling rates according to the DBB. Furthermore, if DBB values and actual positions are captured by different systems (e.g., DBB controller and NC control), both measuring series may have different time offsets and scales, which have to be synchronized afterwards. An approach to solve this synchronization problem is explained in [4].

Due to the arrangement of the fixed and moved ball at the spindle and table of the machine tool, respectively, DBB measurements are affected by all influences on the motion behavior of assemblies and components that are located in the kinematic chain between the two balls. The measuring values contain the superimposed effect of all accuracy relevant influences at the TCP that are currently effective [9]. This mainly includes influences due to geometrical and kinematic errors of the feed-axes and their guidings as well as of the machine tool assemblies. In addition, there are also influences due to position-dependent static deformations resulting from displaced dead weights. The effect due to these influences depends on the measuring poses and the feed-axes involved. There are also inertia influences due to acceleration and deceleration of machine tool assemblies as well as influences due to servo lag in the control loops of the feed-axes. The effect of these influences can be minimized by smooth and jerk-limited measuring motions as well as by feed-forward control. Depending on the previous usage of the machine, thermal influences can also be effective. These influences can be minimized by a short measuring duration, which is an advantage of continuous DBB measurements. Usually there are no influences due to static or dynamic process loads, even though there is considerable research addressing this aspect $[10,11,18]$. 
DBB measurements can only assess the influence of feed-axes that are involved in the measuring procedure. For circular tests according to ISO 230-4 [1] on serial kinematic machine tools, usually only two feed-axes are interpolated simultaneously, since the measuring path is a planar circle. This can provide sufficient assessment results for 2 or 2.5 -axis machining, but is rather limited for the assessment of machining with 5 or 6-axis motions. In general, for a meaningful assessment, all axes that are interpolated simultaneously during machining should be interpolated simultaneously during a DBB measurement as well. This aspect is of particular relevance, if serial kinematic machine tools are compared to parallel kinematic machines or industrial robots, which usually always have to interpolate all feedaxes simultaneously, even when generating a 1-dimensional linear motion of the end effector. There is an 3D enhancement of the ISO 230-4, which not only uses a circle in the $X Y$ plane, but also planar circular arcs in the $X Z$ and $Y Z$ planes, respectively [19]. Meanwhile, this enhancement is also commercially available as "volumetric circularity test". Herein, measuring movements are extended to the third dimension $(Z)$, but they are still planar. That is, the assessment results of such DBB measurements are still limited to 2-3 feed-axes.

Although not considered yet in ISO 230-4 so far, the DBB allows for measuring motions in up to $6 \mathrm{DOF}$ and, thus, for more meaningful assessment results, especially for 5 or 6 axis machining scenarios. On the one hand, the moved ball can be moved around the fixed ball in 3 translational DOF. This results in a possible measuring volume in a sphere shape, whereas its minimum and maximum radius depend on the measuring radius and range of the DBB. On the other hand, rotations around the center of the moved ball in up to 3 additional DOF are possible, as well, which enhances the measuring volume up to 6 DOF. It is bound by the limits of the feed-axes, by collision conditions of the measuring setup, and, in case of parallel kinematic machines, by the limits of passive joints as well as singular configurations. These aspects determine the design of volumetric DBB measuring paths. More on this in Section 5.

\section{DESIGN OF A DBB SYSTEM WITH ENHANCED MEASURING RANGE}

To carry out DBB measurements in up to $6 \mathrm{DOF}$, the $\mathrm{DBB}$ device must meet some requirements. The measuring accuracy must be better than the motion accuracy to be evaluated. Thus, a measuring accuracy of around $1 \mu \mathrm{m}$ or below is targeted. Accordingly, the sensor resolution should be one magnitude higher than the measuring accuracy, hence, a sensor resolution of $0.1 \mu \mathrm{m}$ is targeted. Both requirements imply, that neither clearance nor relevant friction is acceptable in the mechanical parts of the DBB device. Further on, the sensor signals must not be disturbed, and, to minimize Abbe's error, the sensor has to be placed at the connecting line of the ball centers.

Depending on the particular measuring scenario, the measuring range should be large, if possible. Although there are scenarios, which might be satisfied with a measuring range of some $\pm 0.1 \mathrm{~mm}$, there is a lot of scenarios requiring a measuring range of more than $\pm 1 \mathrm{~mm}$. Among them are 6 DOF measuring paths, where superimposed motion errors are larger than the motion errors of a single feed-axis, or the automation of complete measuring cycles, including attachment and detachment of the DBB equipment in the machine, when it is advantageous to have some reserve in measuring range. The same applies to DBB measuring 
paths with positions of non-constant nominal radius or even to different DBB applications, e.g. referencing of tools and workpieces [20]. Thus, in this project measuring ranges of up to $220 \mathrm{~mm}$ have been targeted.

For use in a production environment, the DBB device must be robust, small and lightweight and have a low sensitivity to temperature changes. For data capturing the device must be easily connectable to a PC or the NC control. Therefore, common data interfaces as well as appropriate transfer protocols should be provided. Sampling rates of up to the interpolation cycle time (1000 Hz or above) should be possible.

Although DBB measurements are advantageous compared to other evaluation methods, there are only few providers of DBB devices in the market. The devices sold so far are primarily developed for measurements according to ISO 230-4 and, hence, do not fulfil all the requirements named above. Thus, to be able to investigate possibilities and limits of 6 DOF DBB measurements, an enhanced DBB was developed at the IMD of the TU Dresden (Fig. 5).
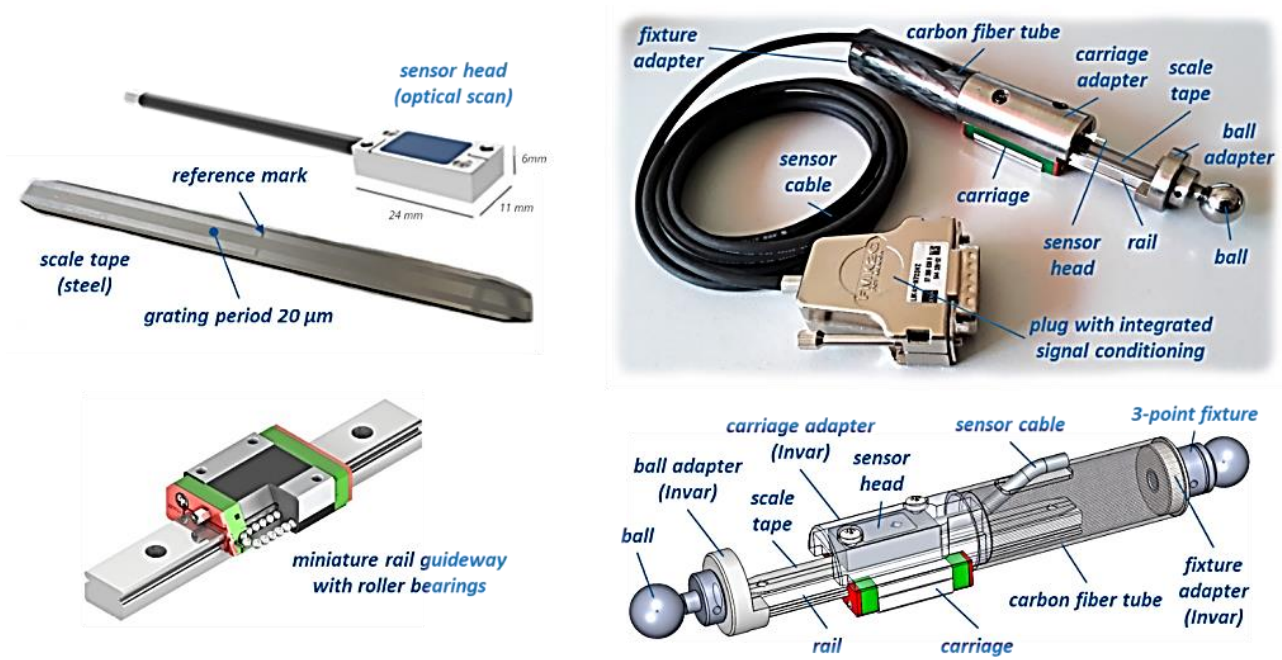

Fig. 5. DBB device with enhanced measuring range, developed at the IMD of the TU Dresden

The core part of the developed DBB device is a linear measuring system that works as an incremental encoder with contactless optical scan. It consists of mainly two components: a miniature sensor head and a scale tape (Fig. 5, top left). The scale tape has a grating period of $20 \mu \mathrm{m}$, which is interpolated 50 times and additionally evaluated 4 times in the counter. This gives a measuring resolution of $0.1 \mu \mathrm{m}$ per increment, while the accuracy of the scale tape is given as $1 \mu \mathrm{m}$ by the manufacturer. The sensor head is already confectioned with a signal cable and a plug and provides integrated signal conditioning. Outside the sensor head differential signals are transferred, so that signal distortions are minimized. From the pins of the plug digital incremental encoder signals can be connected to a suitable counter module.

The relative motion of both DBB components require a precise guiding. For that, a linear guideway of miniature type with roller bearings has been chosen. The scale tape is fixed at the rail of this guideway by a specific adhesive, while the sensor head is mounted into a carriage adapter, that is glued onto the carriage itself. The properties of this guiding have been investigated for various carriages with different length as well as bearing preload. 
A ball adapter connects one rail end to one of the balls, while a carbon fiber tube with a fixture adapter connects the carriage adapter with the magnetic fixture at the other end of the DBB device. All adapters are made of Invar ${ }^{\circledR}$ steel (1.3912, Alloy 36). Compared to conventional steel, it is nearly 10 times less sensitive to temperature changes. The same is said for the carbon fiber tube, which enables a lightweight design and flexible choice of DBB length.

DBB devices with different measuring ranges from $14 \mathrm{~mm}$ up to $220 \mathrm{~mm}$ have been build and investigated at the IMD of the TU Dresden. Different extension adapters allow for an adjustment of the DBB measuring length, e.g. in steps of $50 \mathrm{~mm}$. By using a particular calibration tool that is made of thermally insensitive material (Zerodur ${ }^{\circledR}$ ) and provides accurately known reference lengths (Fig. 6), it is also possible to capture absolute distance values with the DBB. The scale tape contains an additional reference mark, which can be accurately detected by the sensor head, hence, the calibration tool is needed only once, to precisely determine the zero offset of the scale tape.

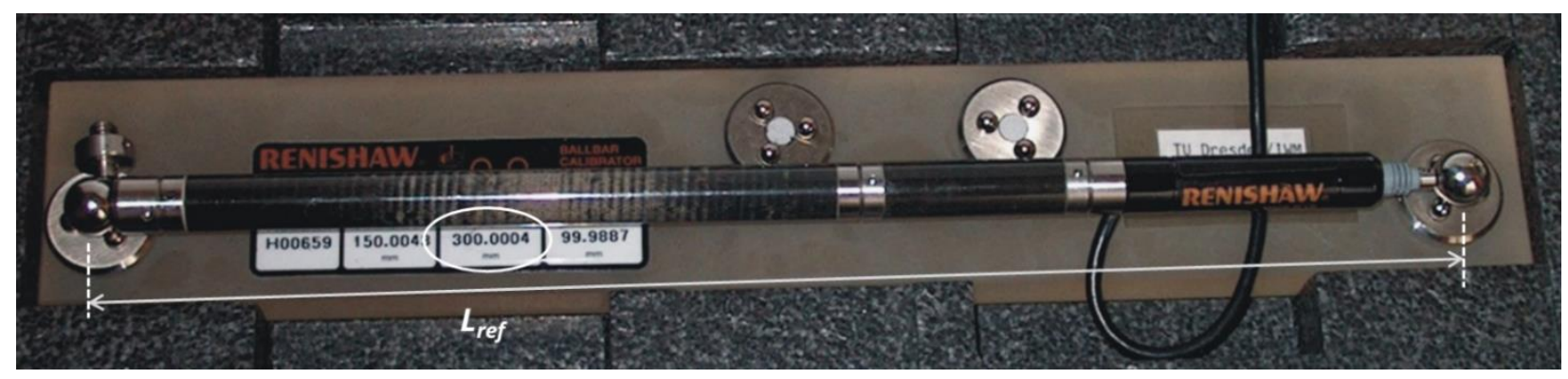

Fig. 6. Calibration tool for absolute radius DBB measurements (Renishaw GmbH)

Two different data interfaces have been provided for data capturing. The first one allows for stand-alone measurements, independent of the machine or control. The DBB is connected to a micro-controller box (Fig. 7, top), developed at the IMD of the TU Dresden. In this box the encoder signals are hardware-counted by an incremental counter chip (up to $32 \mathrm{MHz}$ ), prepared to specific data telegrams (Fig. 7, bottom) and transfered cyclically as a serial data stream to a PC. The PC is connected per USB interface, which also supplies the power for the microcontroller and the sensor. Hence, no additional power supply or batteries are needed.

Two types of cyclic data telegrams are selectable. A short telegramm of 6 Bytes contains the actual measuring value with a resolution of $0.1 \mu \mathrm{m}$ (4 Bytes), the actual state of the sensor system (8 Bits) and a CRC checksum (8 Bits). A long telegram of 10 Bytes additionally provides a time stamp with a resolution of $50 \mu \mathrm{s}$ (4 Bytes) for every measuring value. At the PC side the box is recognized as a virtual serial UART device and can be accessed using standard UART libraries with baud rates of up to $1 \mathrm{Mbit} / \mathrm{s}$. The box can be controlled and configured over serial interface by a set of control commands. Depending on the data processing at the PC side, stable sampling rates of up to $5 \mathrm{kHz}$ are possible in practice.

The second interface allows for control integrated measurements. The DBB device is connected directly to the NC control via a real-time capable fieldbus interface. Fig. 8 exemplarily shows the control integration of the DBB for a TwinCAT-based NC control of Beckhoff. Here an EtherCAT bus coupler (EK1100) and an incremental counter interface 
(EL5101), which also provides the power for the sensor head, were used. This solution allowed for a synchronous sampling of DBB data and actual axis positions in interpolation cycle times of $1 \mathrm{~ms}$ and below. In contrast to the standalone-solution above, no additional data processing due to timing deviations is necessary. However, an according incremental encoder interface needs to be available for the control system at hand and, the control must provide suitable functionality for data capturing, logging and streaming.

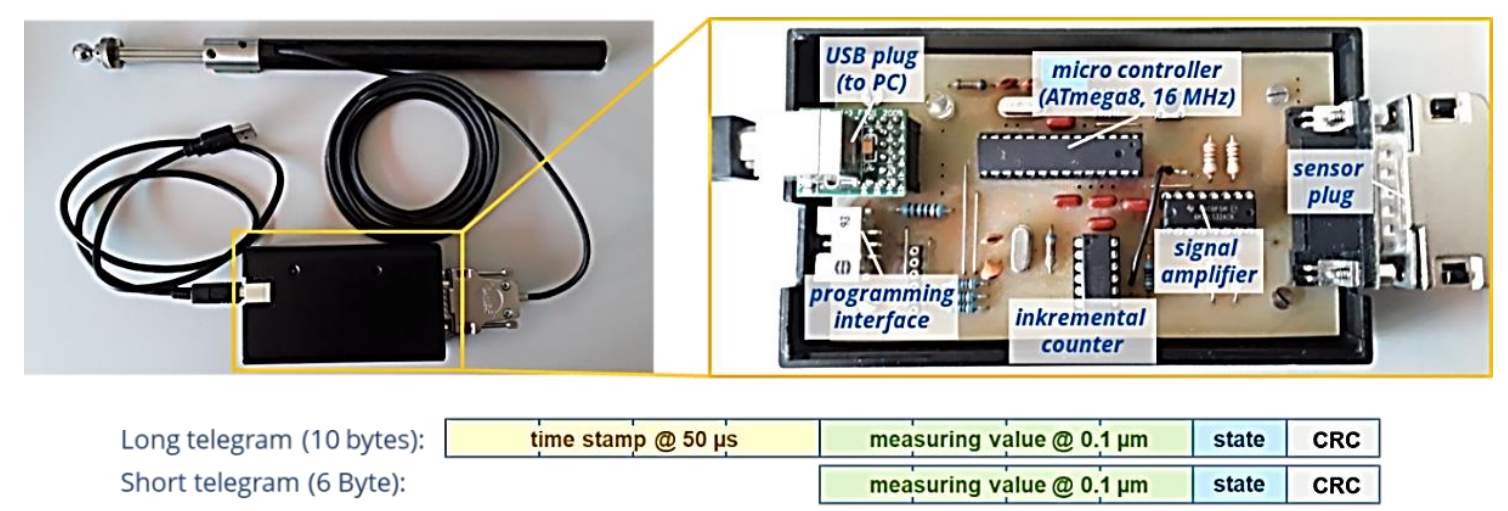

Fig. 7. Data interface for serial USB connection to a PC (above) with provided data protocols (below)

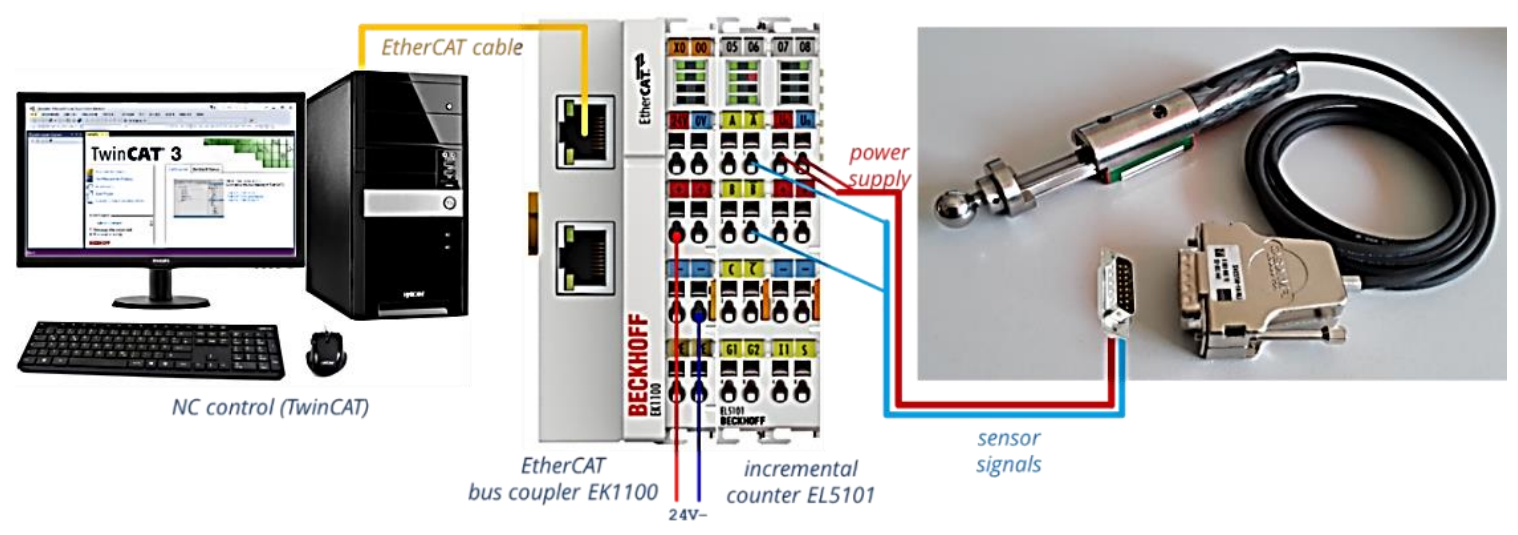

Fig. 8. Data interface of the DBB for integration into a NC control on the example of a Beckhoff TwinCAT system

\section{INVESTIGATION OF MEASURING ACCURACY OF THE DBB}

The accuracy of DBB device is an essential prerequisite for reliable DBB measurements. To investigate the measuring accuracy of different DBB designs, a specific testbed was used (Fig. 9). It contains the DBB device to be investigated (Fig. 9, left), a linear feed-axis with a motion range of $230 \mathrm{~mm}$ (Fig. 9, center) and a 1D laser interferometer (LI) as reference system (Fig. 9, right). The feed-axis includes a slide with two rail guidways. It is driven by a servo motor (Beckhoff) over a toothed belt and a ball screw. The servo motor is controlled by a TwinCAT control system. The moved ball fixture and the LI reflector mirror are mounted on the slide. During setup of the test bed, the laser beam is aligned to run through the centers of the fixed and moved ball in their magnetic fixtures, which renders Abbe's error negligible. 


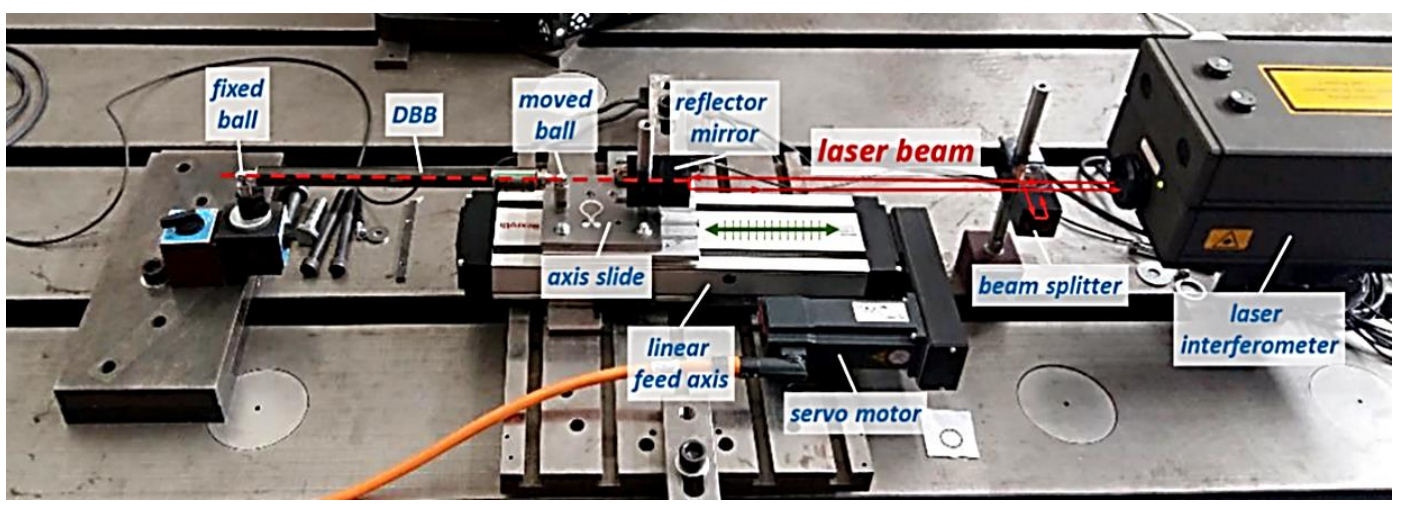

Fig. 9. Test setup for experimental investigation of the measuring accuracy of the DBB system

The measuring procedure for the investigation of the DBB's measuring accuracy was made up of 5 repeated measuring cycles, which ensure a statistical relevance of the measurements and allow for the adjustment of unavoidable thermal effects from measuring data. Each cycle consisted of an intermittent forward and backward motion of the slide over the whole measuring range with around 75 to 150 equidistant steps each, depending on the measuring range. At each single step the motion was stopped for capturing the current measuring value of DBB and LI, respectively. Data capturing was triggered, when the current measuring value remained inside a change window of $+/-0.1 \mu \mathrm{m}$ for a certain time of at least $0.7 \mathrm{~s}$. Thereby, disturbing effects due to mechanical vibrations or control loop influences were minimized and the measured values of the DBB and LI, respectively, can be associated to one and the same physical slide position.

Depending on the measuring range, step count and step pause time, a measuring procedure of 5 cycles took between 20 and 55 minutes. In view of the low measuring resolution of $0.1 \mu \mathrm{m}$, thermal effects due to temperature changes during this time are not negligible. They were recognized as a drift of the raw DBB error of up to several $\mu \mathrm{m}$ over the 5 cycles. Fig. 10 (red) shows this effect on the example of a measuring range of $2 \mathrm{~mm}$ and for all in all 790 measuring steps. However, these thermal effects can be removed from the measurements afterwards.

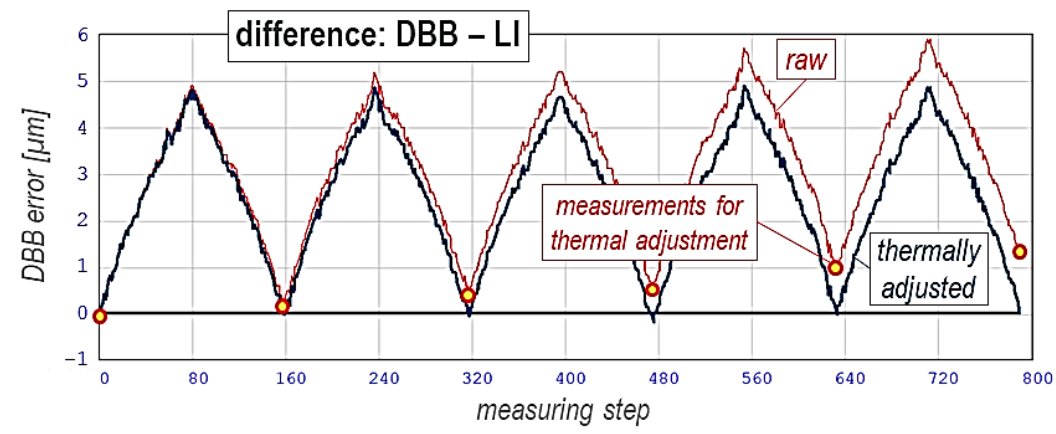

Fig. 10. Thermal adjustment of the DBB error using 6 (out of 790) measuring values

For this purpose, 6 specific raw DBB error values that belong to the same measuring position and motion direction were fitted by a PT1 function, namely the measuring values in the start positions of each of the 5 cycles and the measuring value after the last reverse step 
(Fig. 10, red circles). The fitted PT1 function was then used for the thermal correction for all measured values, according to their respective capturing times. This results in the thermally adjusted DBB error (Fig. 10, blue). By relating it to the corresponding measuring positions, the scaling, linearity and mean variation can also be examined.

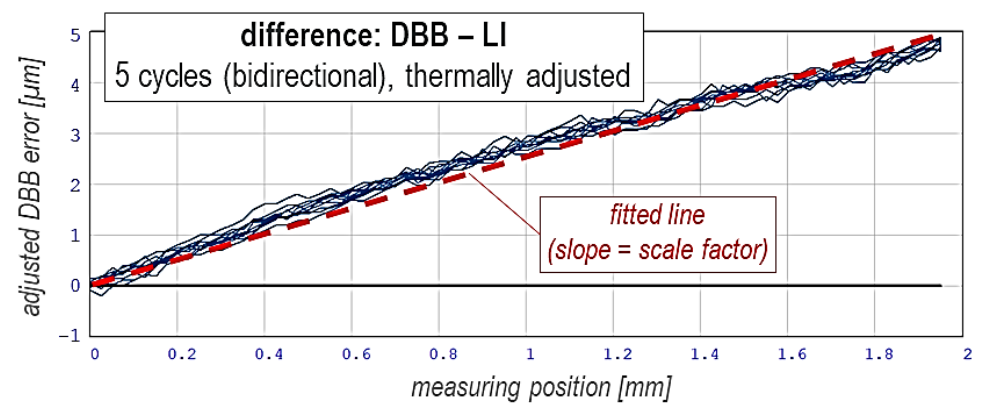

Fig. 11. Linearity, scaling and mean variation of the thermally adjusted DBB error over the measured range $(2 \mathrm{~mm})$

Figure 11 (blue) shows the according plot for all 5 cycles of the example from Fig. 10. Ideally, the values should be arranged close to the horizontal zero line (Fig 11, black). Instead, a significant scaling of around $2.5 \mu \mathrm{m} / \mathrm{mm}$ can be seen. Fitting a line through all the data (Fig. 11, dashed red) shows a quite good linearity of the DBB error, wheras the slope of the fitted line is the scale factor for the DBB sensor system. After scaling, which is equivalent to the difference of the thermally adjusted DBB error to the fitted line, the remaining DBB error results, which, for the example of Fig. 10, is shown in Fig. 12.

These investigations have been made for DBB designs with different measuring ranges $(2 \ldots 150 \mathrm{~mm})$, carriage preloads $(Z \mathrm{~F}, Z 0, Z 1)$ and 2 carriage lengths (contact lengths of 20 or $30 \mathrm{~mm}$ ). In general, the carriages with zero preload (Z0) showed the best (resp. lowest) mean variations and the best linearity. Although preloaded carriages $(Z 1)$ improve the repeatability of the DBB error, they generate significant hysteresis due to the greater friction. The opposite holds for carriages with clearance $(Z \mathrm{~F})$. Furthermore, longer carriages $(30 \mathrm{~mm})$ resulted in better results compared to short ones $(20 \mathrm{~mm})$, even though the difference is only small and rather relevant for larger measuring ranges.

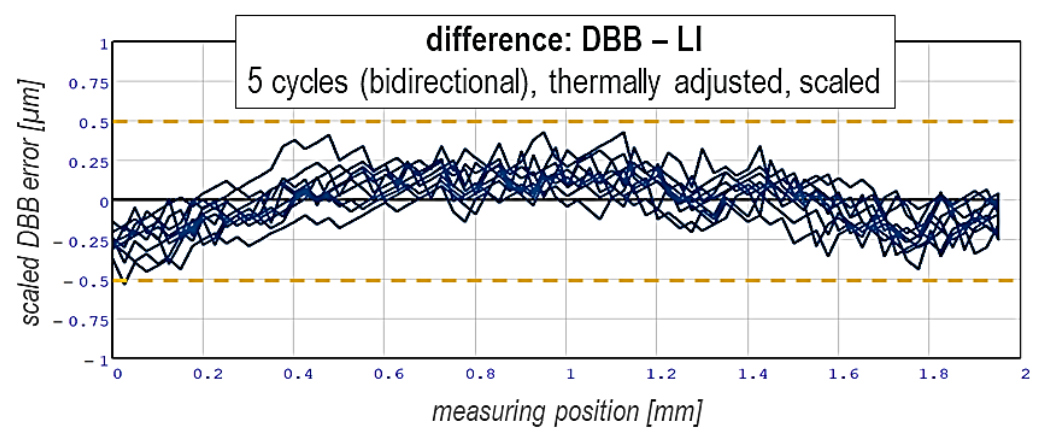

Fig. 12. Remaining DBB error after adjustment and scaling of the measurements over the measured range (2 mm)

An additional measurement was made to compare to a commercially available DBB with a measuring range of $2 \mathrm{~mm}$. Fig. 13 shows exemplary results for a DBB design with up 
to $43 \mathrm{~mm}$ measuring range and a large carriage of zero preload as well as for a DBB design with up to $153 \mathrm{~mm}$ measuring range and a short carriage of zero preload. The comparison was made for strokes of $2 \mathrm{~mm}$ and $5 \mathrm{~mm}$, respectively, in the middle of the respective measuring range. The results in Fig. 13 show the high accuracy of the developed DBB device, which is quite comparable to the commercial system. Even with a larger measuring range of $5 \mathrm{~mm}$, the resulting DBB error always remains below $1 \mu \mathrm{m}$.

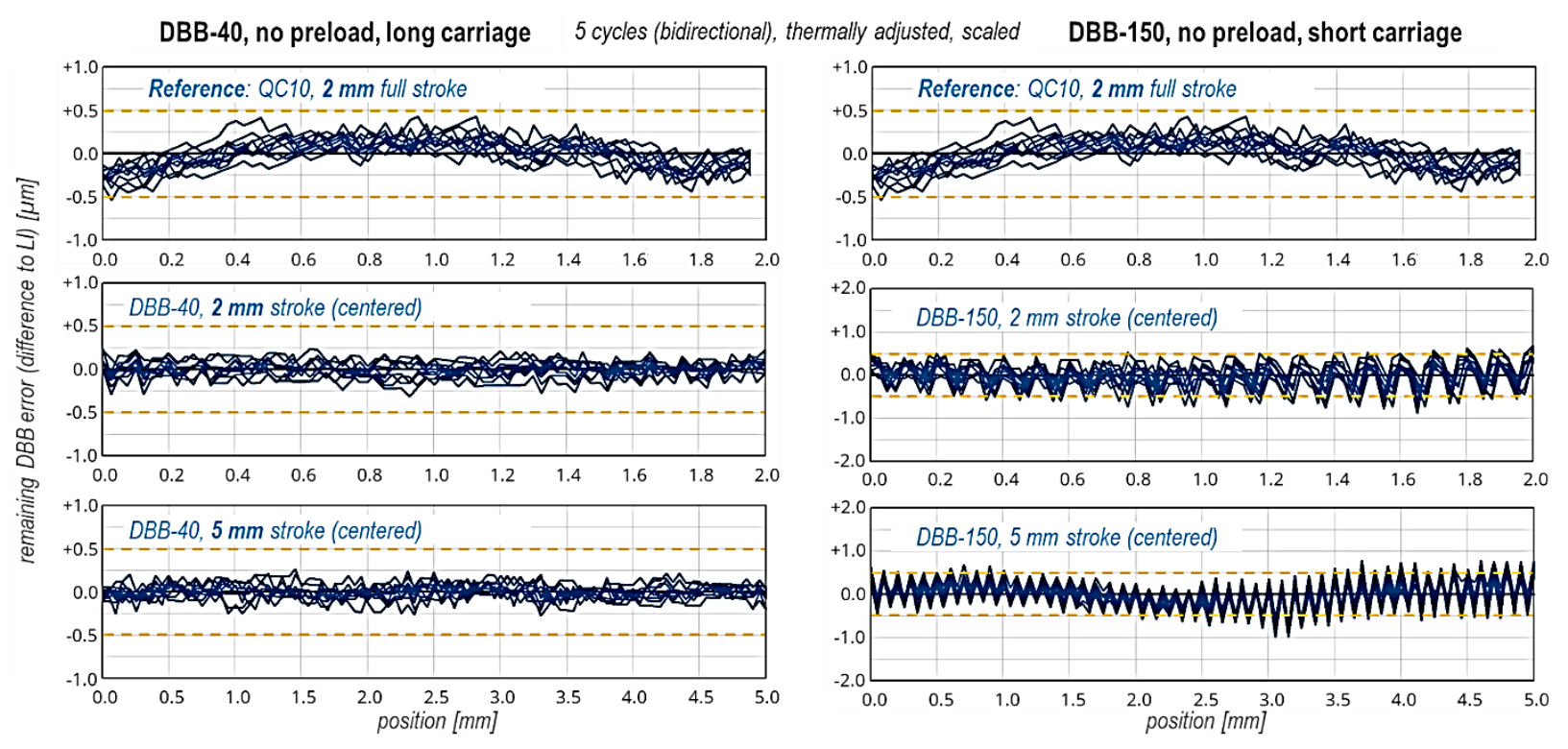

Fig. 13. Exemplary results of the experimental evaluation of measuring accuracy for strokes of 2 and $5 \mathrm{~mm}$, compared to a commercial DBB device (above)

The accuracy achieved with the developed DBB device is mainly based on the accuracy of the incremental sensor system used. Thermal effects obviously have a considerable influence. To achieve a higher measuring accuracy either the thermal conditions during the measurements must be kept as constant as possible or additional temperature information should be captured during the measurement and taken into account. While the former can be realized to only a limited extent in practice under shop floor conditions, the latter is associated with a further development of the DBB and the post-processing of raw measuring data.

The results show, that the developed DBB device meets the accuracy requirements for the assessment of NC machines outlined at the beginning of Section 3. The design is robust and suitable for measurements under shop floor conditions. Material costs including the controller box remained in a range of about 1000 Euro. Thus, it can be considered to be quite inexpensive compared to other commercially available measuring devices that can provide a similar measuring range, accuracy and resolution.

\section{DESIGN OF VOLUMETRIC MEASURING PATHS}

In contrast to simple planar circles measuring paths with $6 \mathrm{DOF}$ have a greater complexity due to the additional DOF's, and more complex requirements that have to be met. 
Primarily, a majority of the error components of the machine tool should be actually effective on the measuring path. Thus, one of the most inaccurate motion paths is searched for. In addition, the measuring path must:

- be continuous in all 6 DOF in order to facilitate quick measurements of short duration,

- take into account all motion limits and collision conditions,

- be formulated as a valid NC program for the particular NC control at hand.

Furthermore, it should be jerk-limited to avoid unwanted excitation of the machine structure during DBB measurements, unless this effect is to be explicitly analysed.

To find a suitable 6 DOF DBB measuring path the following method was developed, based on the four-step approach according to Fig. 14. Mathematical details of this approach have been already published in [21] and are left out here, for this reason.
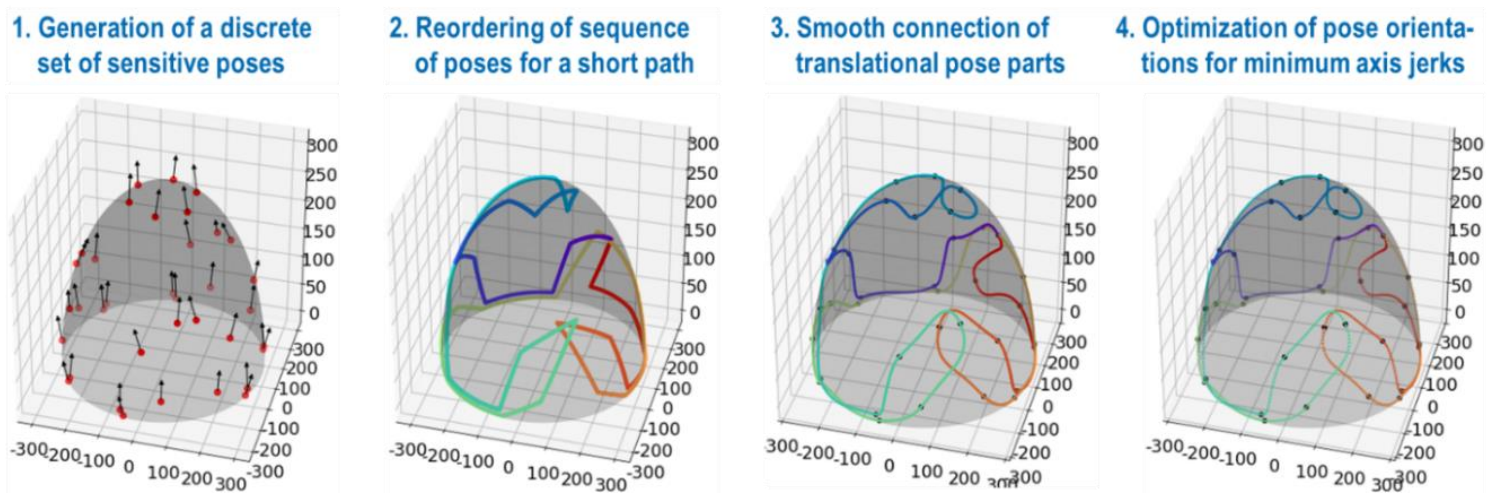

Fig. 14. Generation of a suitable 6D path with constant nominal radius for DBB measurements

\subsection{GENERATION OF A DISCRETE SET OF SENSITIVE POSES}

A set of discrete 6 DOF poses in the workspace is generated, that reach an extremal value for a specific evaluation criterion depending on kinematic structure and evaluation scenario. A typical example of such a criterion is the sensitivity of DBB values to single kinematic error sources, which holds for the most or least accurate poses in the workspace under consideration (e.g. covered by a sphere). Other criteria could be tailored to specific manufacturing tasks, e.g. considering particular error sources or orientational errors only.

To do this, a simulation model is required as a prerequisite, that contains the kinematic structure including all relevant error sources and calculates the corresponding DBB measuring value depending on a pose in the workspace and the error values. Based on this, a stochastic optimization procedure was developed. It defines the criteria to be considered in its objective function. In addition, motion limits of feed-axes and passive joints (in case of parallel kinematics) must not be exceeded by any of the poses. Collisions of the DBB and machine components and singularities have to be avoided in the measuring path as well. These aspects are regarded by according penalty functions, extending the objective function.

In principle, the number of discrete poses can be chosen freely. On the one hand, it should be a large number for a good quality of the measuring path. On the other hand, the more poses are used the more calculation time is required and the measuring path becomes more complex and possibly longer. Although the optimal choice of the number 
of poses requires further investigation, promising results have been achieved so far if the number of poses corresponded at least the number of single error sources or higher.

\subsection{REORDERING OF SEQUENCE OF POSES FOR A SHORT PATH}

For a continuous motion the discrete poses have to be connected to form a measuring path. In order to achieve a possibly short path length and motion duration, respectively, the poses need to be sorted appropriately. To do this, a "Traveling Salesman Problem" (TSP) must be solved. The "Concorde TSP Solver" tool was used for this, which is freely available in the internet [22]. Besides the path length also the feasibility and the collision conditions of the intermediate poses on the connection paths were considered in the TSP's cost matrix.

\subsection{SMOOTH CONNECTION OF TRANSLATIONAL POSE PARTS}

The resulting translational path of step 2 is a spherical polygon that has kinks at the single poses. This would lead to discontinuous motions with speed reduction at these poses. Therefore, a smooth path is generated, which leads through every single pose and runs C1-steadily (without discontinuity in speed) in the translatory components. For this, the translational tangents at each pose are determined and optimized, so that the resulting path lies completely in the upper hemisphere, to avoid any possible collision between moving ball and machine table.

\subsection{OPTIMIZATION OF POSE ORIENTATIONS FOR MINIMUM AXIS JERKS}

Afterwards the orientational components of the poses are calculated. For this purpose, a larger number of poses of around $1 \mathrm{~mm}$ distance is interpolated from the smoothed path of step 3, whereas the orientations of the intermediate poses are initially calculated by spherical linear interpolation [23] using a sinusoidal interpolation parameter. For each of these poses the orientation is then varied by a stochastic optimization method. The objective function was designed for minimizing the maximal occurring jerks of the feed-axes, while the feasibility and collision conditions have been considered by additional penalties.

Based on the poses obtained from these four steps, finally an NC program can be generated, usually in G-Code format according to DIN 66025/ISO 6983. As a sequence of G1 blocks the poses form polygon vertices or spline nodes, depending on the supported functionality of the NC control type at hand. Even though G-Code is standardized [24], control-specific dialects of G-Code are also common, which must be taken into account when generating the NC programs. The whole measuring procedure can be further automated with additional motion sequences in the NC program for the setup of the fixed ball and the DBB in the machine.

The algorithms used in the four steps have been implemented in python to be universally applicable. However, some machine specific informations have to be provided, additionally: an appropriate kinematic model, kinematic as well as dynamic limits of feed-axes and passive joints (in case of parallel kinematics), collision conditions in the machine workspace and the NC control type. 


\section{MEASUREMENT RESULTS}

On the example of a hexapod in the test field of the IMD at the TU Dresden, three different DBB measuring paths were examined in view of the derivable statements. The measuring paths were:

- a planar circular path (2D) in the $X Y$ plane,

- a suboptimal 6D measuring path from [12] with only small orientation angle values,

- a 6D measuring path optimized with the developed method.

The measuring radius was $300 \mathrm{~mm}$ in each case. The measurements were carried out one after another, so that the error state of the machine was essentially identical for the three paths. The results are shown in Fig. 15:
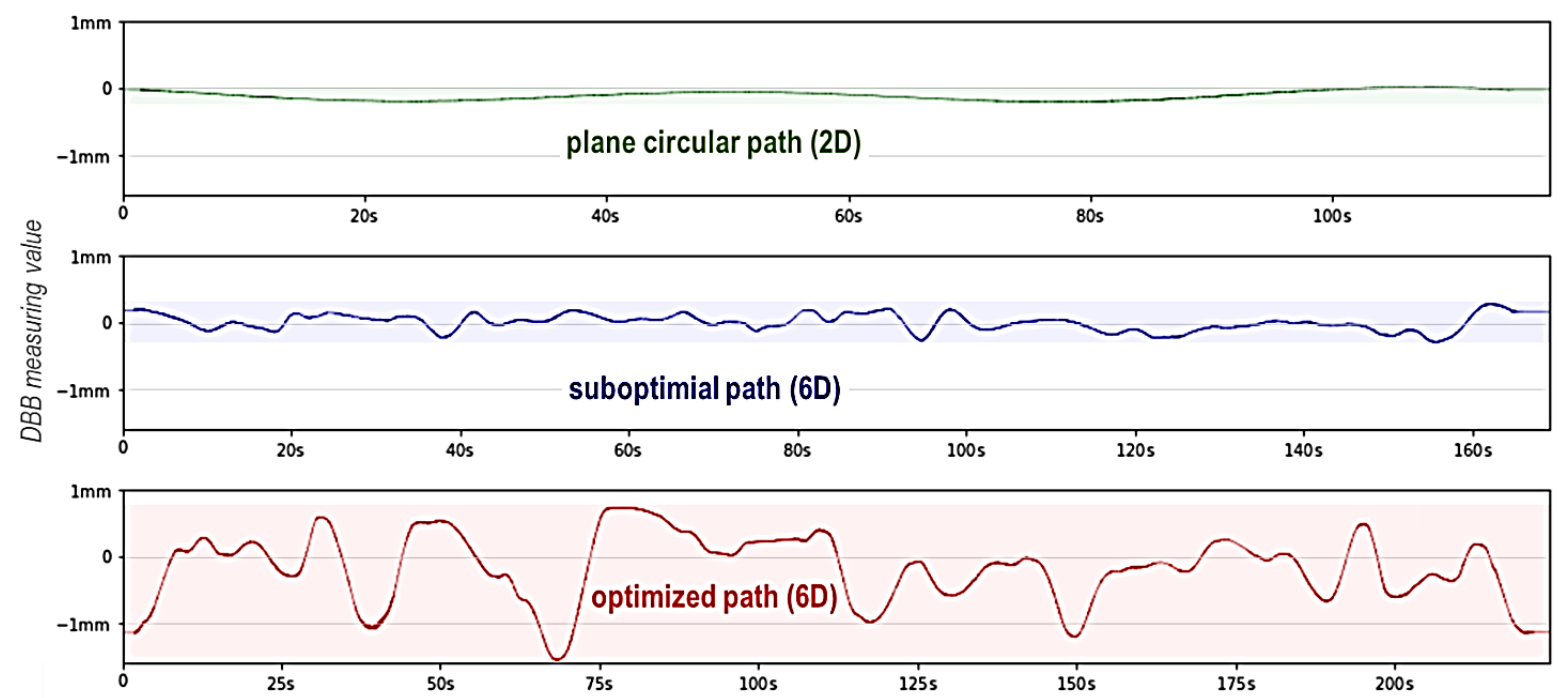

Fig. 15. DBB measurements at one and the same machine with different measuring paths of $\mathrm{R}=300 \mathrm{~mm}$

Using the difference between the span of the DBB values as a comparison criterion, it ranges from $0.2268 \mathrm{~mm}$ for the 2D circular path, over $0.5863 \mathrm{~mm}$ for the suboptimal path to $2.3153 \mathrm{~mm}$ for the optimized path. Related to the planar circular path, this is an increase by factor 2.6 for the suboptimal and by factor 10.2 for the optimized path. More extended statements can be derived by means of a Fourier analysis of the DBB values, see Fig. 16:

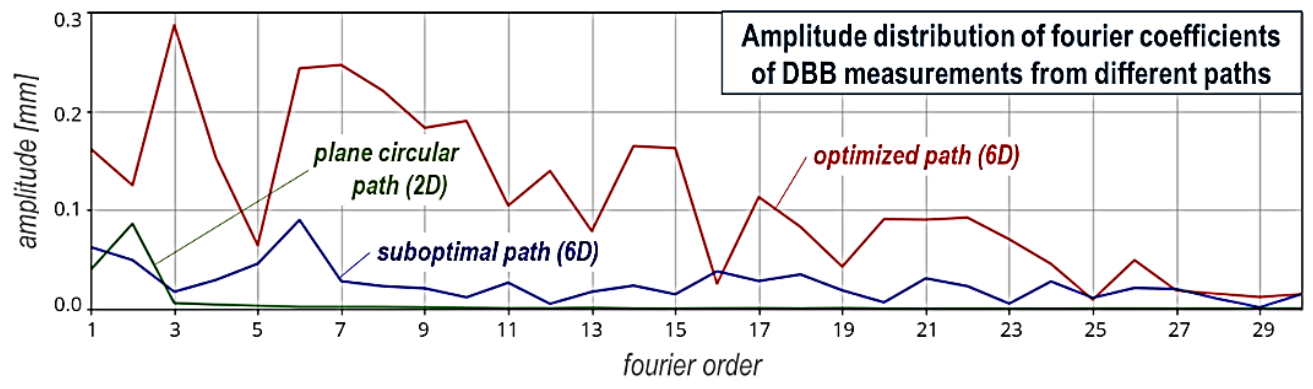

Fig. 16. Amplitude distribution of Fourier coefficients of the DBB values for the 3 different measuring paths 
The level of the amplitudes and the number of Fourier coefficients with amplitudes above a certain limit can be interpreted as a measure of information content (entropy) of the DBB values. In comparison to the other measuring paths, the optimized measuring path here shows significantly higher amplitudes in more Fourier coefficients. Hence, it provides more information about motion errors and is more sensitive to it.

\section{SUMMARY AND OUTLOOK}

The paper presents an alternative approach for the evaluation of the volumetric motion accuracy of machine tools by using a cost-efficient Double-Ballbar and optimized 6 DOF measuring paths. It is particularly suitable for measurements in an operational state of the machine, under shop floor conditions and with minimum machine downtime of up to only some minutes. Hence, fast performance tests of the volumetric accuracy can be carried out easily and more frequently in operational pauses, which allows an economical accuracy monitoring by comparing the measured values to a reference state of the machine tool at short intervals.

The design of a DBB system with enhanced measuring range of up to $220 \mathrm{~mm}$ and two different interfaces for stand-alone and control integrated data acquisition was explained. The experimental setup and the measuring procedure for the analysis of the measuring accuracy of the developed DBB was shown. Depending on the measuring stroke, the developed DBB can reach an accuracy of $1 \mu \mathrm{m}$ as well as a repeatability of below $1 \mu \mathrm{m}$, which is comparable to DBB systems available in the market, although there are only few providers, so far.

Furthermore, a design method for optimized 6 DOF measuring paths, based on a fourstep approach, was outlined. Due to the greater complexity of the design problem, an efficient generation of NC programs for volumetric DBB measurements requires suitable software support. The algorithms have been implemented in python. Potentials of optimized 6 DOF measuring paths have been demonstrated on the example of 3 measuring paths of different dimension and complexity. Compared to the other paths, the optimized 6 DOF measuring path showed significantly better results in view to sensitivity and information content of the DBB values measured along this path.

Further research is advisable in view to a more efficient generation of 6 DOF measuring paths, to the considering of additional kinematic structures, to an efficient automation and cross-check of the whole measuring procedure, to the design of tailored measuring paths for specific statements (e.g. the feasibility for specific machining tasks) or even to the identification of particular error parameters and kinematic calibration procedures, respectively.

\section{ACKNOWLEDGEMENTS}

The authors want to thank the German Research Foundation (DFG) for their kind support in the project IH 124/3-1 with the title "Measurement and evaluation of the volumetric accuracy of multi-axis machine tools under operational conditions". 


\section{REFERENCES}

[1] ISO 230-4, 2005, Test code for machine tools Part 4: Circular Tests for Numerically Controlled Machine Tools.

[2] NAFI A., LOS A., MAYER J.R.R., 2010, Axis Location and Scale Factors Estimation for Three-Axis Machines from Periodic Performance Checks with Laser Distance Measurements - Risks and Opportunities, Journal of Machine Engineering, 10/4, 89-99.

[3] NI Y., LIU X., ZHANG B., ZHANG Zh., LI J., 2018, Geometric Error Measurement and Identification for Rotational Axes of a Five-Axis CNC Machine Tool, Journal of Mechanical Engineering, 64/5, 290-302.

[4] MILLER J.E., LONGSTAFF A.P., PARKINSON S., FLETCHER S., 2017, Improved Machine Tool Linear Axis Calibration Through Continuous Motion Data Capture, Precision Engineering, 47, 249-260.

[5] DASSANAYAKE K.M.M., YAMAMOTO K., TSUTSUMI M., SAITO A., MIKAMI S.H., 2008, Simultaneous Five-Axis Motion for Identifying Geometric Deviations Through Simulation in Machining Centers with a Double Pivot Head, Journal of Advanced Mechanical Design, Systems, and Manufacturing, 2/1, 47-58.

[6] IHLENFELDT S., 2012, Redundante Werkzeugmaschinenstruktur für die Komplettbearbeitung im Großwerkzeugbau: modellbasierter Systementwurf und Prototyp, Ph.D. Thesis, TU Chemnitz.

[7] IHLENFELDT S., WABNER M., FRIESS U., TEHEL R., 2015, Berücksichtigung des positionsabhängigen Maschinenverhaltens innerhalb der Simulation: Dynamische Simulation holistischer FEM-Modelle, wt Werkstattstechnik Online, 105/5, 275-284.

[8] DAHLEM P.H., MONTAVON B., PETEREK M., SCHMITT R.H., 2018, Enhancing Laser Step Diagonal Measurement by Multiple Sensors for fast Machine Tool Calibration, Journal of Machine Engineering, 18/2, 64-73.

[9] KAUSCHINGER B., 2006, Verbesserung der Bewegungsgenauigkeit an einem Hexapod einfacher Bauart, Ph.D. Thesis, TU Dresden.

[10] ARCHENTI A., OSTERLIND T., NICOLESCU C.M., 2011, Evaluation and Representation of Machine Tool Deformations, Journal of Machine Engineering, 11/4, 105-117.

[11] ARCHENTI A., NICOLESCU M., CASTERMAN G., HJELM S., 2012, A New Method for Circular Testing of Machine Tools Under Loaded Condition, 5th CIRP Conference on High Performance Cutting 2012, Procedia CIRP, 1, 575-580.

[12] SZATMÁRI S.Z., 2007, Kinematic Calibration of Parallel Kinematic Machines on the Example of the Hexapod of Simple Design, Ph.D. Thesis, TU Dresden.

[13] BRYAN J.B., 1982, A Simple Method for Testing Measuring Machines and Machine Tools, Precision Engineering 4/2, 61-69, 125-138.

[14] BRYAN J.B., 1982, Telescoping Magnetic Ball Bar Test Gage, US Patent 4,435,905, filed March $15,1982$.

[15] KAKINO Y., 1986, Method and Device to Measure Motion Errors of NC Machine Tools, European Patent Application EP 0258471 A1, date of filing 01.09.1986.

[16] WECK M., BRECHER C., 2005, Werkzeugmaschinen 5 Messtechnische Untersuchung und Beurteilung, dynamische Stabilität, CRC Press; $2^{\text {nd }}$ Edition, ISBN-13: 978-08-2475-888-2.

[17] YANG S.-H., LEE H.-H., LEE K.-I., 2018, Face- and Body-Diagonal Length Tests using a Double Ball-Bar for Squareness Errors of Machine Tools, Int. J. of Precision Engineering and Manufacturing, 19/7, 1039-1045.

[18] DAPERO A., ARCHENTI A., 2015, Identification and Analysis of Linked System's Dynamic Accuracy - Proposal of a Measurement Approach and Instrumentation, Journal of Machine Engineering, 15/3, 91-104.

[19] GROSSMANN K., WUNDERLICH B., 2001, Preiswerte Genauigkeit am Hexapod - Strukturmodellbasierte Fehlerkorrektur, Zeitschrift für wirtschaftlichen Fabrikbetrieb, ZwF 96/5, 257-261.

[20] GROSSMANN K., KAUSCHINGER B., 2008, Räumliche Referenzierung an Werkzeugmaschinen mit dem Double-Ball-Bar, Zeitschrift für wirtschaftlichen Fabrikbetrieb, ZwF, 103/3, 104-110.

[21] ZHOU R., KAUSCHINGER B., IHLENFELDT S., 2020, Path Generation and Optimization for DBB Measurement with Continuous Data Capture, Measurement, 155, 107550, 10.1016/j.measurement.2020.107550.

[22] http://www.math.uwaterloo.ca/tsp/concorde.html

[23] SHOEMAKE K., 1985, Animating Rotation with Quaternion Curves, SIGGRAPH 1985, July 22.

[24] DIN 66025: https://www.beuth.de/de/norm/din-66025-2/1394941, 20.12.2019. 